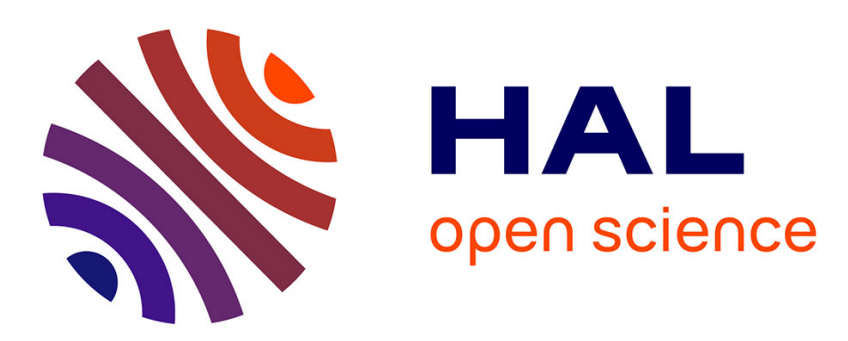

\title{
On the micro-heterogeneous structure of neat and aqueous propylamine mixtures: a computer simulation study
}

Martina Požar, Aurélien Perera

\section{- To cite this version:}

Martina Požar, Aurélien Perera. On the micro-heterogeneous structure of neat and aqueous propylamine mixtures: a computer simulation study. Journal of Molecular Liquids, 2016, 10.1016/j.molliq.2016.12.005 . hal-01414280

\section{HAL Id: hal-01414280 \\ https: / hal.sorbonne-universite.fr/hal-01414280}

Submitted on 12 Dec 2016

HAL is a multi-disciplinary open access archive for the deposit and dissemination of scientific research documents, whether they are published or not. The documents may come from teaching and research institutions in France or abroad, or from public or private research centers.
L'archive ouverte pluridisciplinaire HAL, est destinée au dépôt et à la diffusion de documents scientifiques de niveau recherche, publiés ou non, émanant des établissements d'enseignement et de recherche français ou étrangers, des laboratoires publics ou privés. 


\title{
On the micro-heterogeneous structure of neat and aqueous propylamine mixtures: a computer simulation study
}

\author{
Martina Požar ${ }^{1,2}$ and Aurélien Perera ${ }^{1 *}$ \\ ${ }^{1}$ Laboratoire de Physique Théorique de la Matière Condensée (UMR CNRS \\ 7600), Université Pierre et Marie Curie, 4 Place Jussieu, F75252, Paris cedex 05, \\ France \\ ${ }^{2}$ Department of Physics, Faculty of Sciences, University of Split, Rudera \\ Boškovića 37, 21000, Split, Croatia
}

\begin{abstract}
The micro-heterogeneous structure of neat and aqueous propylamine is examined through computer simulations. Neat propylamine is found to have a pre-peak in the nitrogen-nitrogen structure factor, due to the presence of branched chain-like aminogen clusters. Aqueous propylamine mixtures are found to be micro-segregated at all amine concentrations. Both the waterwater and the amine nitrogen-nitrogen structure factors show the caracteristic domain pre-peaks in their respective moderate to high contents. The amine cluster pre-peak, still visible at high amine content, disappears into the domain pre-peak at lower amine contents. Interestingly, water domains forms linears clusters for water concentrations below equimolar. We discuss the specificity the amine brings to the nature of the water clustering as compared with other type of solutes. In particular, we find that the KirkwoodBuff integrals are quasi-ideal in the amine mole fraction range $0.3<x<1$, which we interpret as being consistent with the linear water clusters observed in this range, and which act as individual supra-molecular entities. We conjecture that such cluster shapes are consistent with the existence of a lower critical solution temperature for this system as well as other aqueous amines.
\end{abstract}

\footnotetext{
*corresponding author (aup@lptmc.jussieu.fr)
} 


\section{Introduction}

Amines play important role in industrial $[1,2,3]$ and pharmacological $[4,5]$ applications, but also in biological processes $[6,7,8,9]$. They bear an interesting resemblance with mono-ols in that they form hydrogen bonds through the lone pairs of the nitrogen atom. Since alcohols form micro-heterogeneous mixtures with water [10], one equally expects amines to behave similarly. However, the $\mathrm{NH}$ group of amines has a 2 donor - 1 acceptor, instead of the 1 donor -1 acceptor structure of $\mathrm{OH}$ hydroxyl group of linear alcohols. How does this difference affects the micro-structure of neat amines and aqueous amine mixtures? For example, it is well known that linear alcohols form chain-like cluster structures, which produce a pre-peak in the oxygen-oxygen structure factor and the total scattered intensity as seen through neutron or Xray diffraction experiments [11, 12]. The existence of two donor sites for amines would favour branched chains. How this particularity could affect amines, as compared to linear mono-ols? Lower amines such as methylamine and ethylamine are gases under ambient conditions, while methanol and ethanol are liquid. This fact indicates that, despite their double hydrogen bonding structure, amines have less hydrogen bonding capabilities than the corresponding mono-ols. In order to appreciate this difference, it is instructive to look at the classical force field representation of the hydrogen bonding through their Coulomb contributions as partial charges on the oxygen, nitrogen and hydrogen atoms. The Optimized Potential for Liquid Simulation (OPLS) [13] force field attributes for propylamine the valence $z_{N}=-0.9$ to the nitrogen atom and valence $z_{H}=+0.360$ to the hydrogen atom. Similarly, it attributes for 1 propanol the valence $z_{O}=-0.7$ to the oxygen atom and the valence $z_{H}=+0.435$. Both sets of values are about the same order of magnitude. Therefore, it seems reasonable to consider that it is the topology of the amidogen $\mathrm{NH}_{2}$ group of the amine molecules which does not allow as much hydrogen bonding as the hydroxyl $\mathrm{OH}$ group of the alcohol molecules. In particular, the proximity of the two hydrogen atoms next the nitrogen atom may hinder efficient hydrogen bonding. This fact may have its importance in the cluster formation and micro-heterogeneous domain formation under mixing conditions. For example, it is experimentally known that aqueous amine mixtures tend to have a lower critical solution temperature (LCST)[14]. This is in variance with aqueous mixtures of small alcohols, which generally tend to have an upper critical solution temperature under ambient pressure conditions, such as aqueous 1 butanol (at 397.9K [15]) and 2butanol (at 390.35K [16]), for example. It is tempting to associate this difference between the two types of mixtures to the underlying topological properties of the two types of solute molecules. For example, in terms of the degree of hydrogen bonding, alcohols may tend to form a more coherently bonded liquid than amines, under ambient conditions, which could explain the temperature dependance of the way these liquids mix with wa- 
ter. In this context, it is interesting to ask how this difference would affect the type of micro-heterogeneity these mixtures have.

Amines have not been studied by computer simulation as much as alcohols, as can be seen by the scarcely available force fields[17], other than the usual Gromos[18, 19] and OPLS[20, 21] transferable force fields. Kusalik et al.[22] have studied methylamine and aqueous methylamine. Lachet et al.[17] have studied a large variety of alkanolamines and their aqueous mixtures, and have proposed a new force field in order to obtain better excess enthalpies.

The principal objective of this paper is to use our previous studies about microheterogeneity to explore for the first time the micro-heterogeneous structure of both neat and aqueous propylamine. This study is a continuation of previous studies where we have shown that the structure of the solute molecule greatly affects the way water segregated domain forms. For example, in aqueous alcohols, water tends to form bulky domains $[23,24]$. In aqueous DMSO, water forms chain-like cluster domains [25]. In both cases, the existence of such segregated domains has two distinct type of impact on the structural and thermodynamical properties of such mixtures. Firstly, a domain pre-peak is found in the oxygenoxygen structure factor $S_{O O}(k)$ at a k-vector intermediate betwen $k=0$ and the main peak about $k=2 \pi / \sigma_{W} \approx 2 \AA^{-1}$, the latter which corresponds to the the diameter of the water molecule $\sigma_{W} \approx 3.1 \AA$. The exact position of this peak, as well as its height, depends on the bulkiness of the segregated water domains. Secondly, the concentration fluctuations depend on the nature of the domains, and tend to be rather high for domain that are fuzzy (such as in tbutanol-water) or with large polydispersity (such as in benzene-ethanol), and small for domains with well stabilized shapes (such as aqueous-DMSO) [26]. Concentration fluctuations, defined as $C_{i j}=<N_{i} N_{j}>-<N_{i}><N_{j}>$, where $N_{i}$ is the number of molecules of species $i$ in a given volume $V$, are related to the structure factors $S_{i j}(k=0)$ through the relation [27]

$$
S_{i j}(k=0)=\frac{<N_{i} N_{j}>-<N_{i}><N_{j}>}{\sqrt{<N_{i}><N_{j}>}}
$$

In a recent work [28], we have argued that segregated water domains were made of groups of smaller water clusters, the latter which were the elementary entity behind micro-segregation. This way of looking at micro-segregation opens new perspectives with respect to general physical chemistry, mainly for a new interpretation of phenomena such as, for example, the hydrophobic effect [29, 30, $31,32]$, as well as the role of water in the biophysical context[33, 34]. The way water forms clusters and the way these clusters group themselves into larger domains or not, seems to depend very strongly on the nature of the solute[26]. For these reasons, it is interesting to explore particular solutes, in order to understand 
the common mechanism behind the behaviour of water. We have chosen propylamine since it is miscible with water at all proportions, and lower alkylamines are less miscible.

In the present paper, we will adopt the same type of approach that we have considered in our previous ones, namely with respect to force fields. While it is important to represent force fields properly in order to reproduce best target physico-chemical properties, we adopt the view that these considerations do not affect qualitatively the micro-segregation property of corresponding aqueous mixtures. In other words, while peculiar details of micro-heterogeneity, such as the pre-peak height and position, for example, might be as sensitive to force field details as other physical properties such as excess enthalpy or density, for example, its existence is essentially robust to such details. This approach has been particularly successful in allowing to predict the correct experimental Kirkwood-Buff integrals $(\mathrm{KBI})$, despite strong differences in the short range correlations corresponding to different force fields, for example in the case of aqueous tbutanol [23] and aqueous acetone [35]. This is an important consideration since the KBI are expressed in terms of volumetric quantities as well as chemical potential derivatives, which are all sensitive to force field details. Since the KBI are representative of the nature of concentration fluctuations, these findings imply an interesting universality relationship between the robustness local molecular organisation, such as clustering or micro-segregation, and force field details.

\section{Model, Simulation and Theoretical details}

All simulations were performed with the program package Gromacs [36]. The forcefield Gromos53a6 [18] was used to model propylamine, while SPC/e [37] was chosen for water. Apart from neat propylamine, we have simulated the propylamine-water mixture over a range of concentrations, typically for propylamine mole fractions of $0.2,0.5$ and 0.8 . The box of neat propylamine contained 2048 particles, corresponding to the box length of $64.7 \AA$. As for the propylaminewater mixture, system sizes of both $\mathrm{N}=2048$ and $\mathrm{N}=16000$ were considered, yielding an average box size of $54.6 \AA$ and $108.4 \AA$, respectively. The force field for 1propanol, used for comparison purpose, was taken from the OPLS series[13] and a system of $\mathrm{N}=2048$ molecules was simulated.

The systems were simulated in the isobaric-isothermal (constant NpT) ensemble, at the temperature of $\mathrm{T}=300 \mathrm{~K}$ and pressure $\mathrm{p}=1$ bar. Those conditions were achieved with the Nose-Hoover thermostat[38, 39] and Parrinello-Rahman barostat[40]. We followed the same procedure for every simulation. Packmol[41] was used to obtain the initial configurations of the systems from the pdb files of each molecule. After energy minimization, the systems were equilibrated in the 
NVT and then NpT ensemble, for a total of 1 ns. The following production runs lasted $4 \mathrm{~ns}$, in order to sample at least 2000 configurations for calculating site-site correlation functions $g_{a_{i} b_{j}}(r)$, where $a_{i}, b_{j}$ represent any two atomic sites on the molecules and $\mathrm{i}, \mathrm{j}$ correspond to the species index.

On top of site-site correlation functions, we calculated the site-site structure factors $\mathrm{S}(\mathrm{k})$, defined as [27]:

$$
S_{a_{i} b_{j}}(k)=S_{a_{i} b_{j}}+\rho \sqrt{X_{i} X_{j}} \int d \vec{r}\left[g_{a_{i} b_{j}}(r)-1\right] \exp (\overrightarrow{i k} \vec{r})
$$

where $\rho$ is the number density and $x_{i}$ the mole fraction of species $i$. The values we report here are obtained by direct Fourier transformation of the correlation functions $g_{a_{i} b_{j}}(r)$ by standard numerical methods[42]. As for displaying the structure factors, we follow the same convention as in our previous works and replace $\delta_{a_{i} b_{j}}$ with 1 in the figures.

Cluster distribution probabilities are calculated within the Gromacs program.

The Kirkwood-Buff integrals[43], mentioned in the previous section, are defined as:

$$
G_{i j}=\int d \vec{r}\left[g_{a_{i} b_{j}}(r)-1\right]
$$

where $g_{a_{i} b_{j}}(r)$ is the site-site correlation function. Since these integrals can be related to macroscopic thermodynamic quantities, they depend only on the species index, and not on the atomic ones[27]. As explained in our previous works[35, 44], these integrals cannot be evaluated directly from correlation functions obtained in the simulation, since these latter are affected at long range by the concentration fluctuations inside the simulation box, and do not tend to the expected asymptote, as was shown by Lebowitz and Percus[45]. We have developed a procedure to account for this problem[44, 26].

The KBI are defined from the relations derived from the KB theory [43, 46, 47]

$$
G_{i j}=\left[k_{B} T \kappa_{T}-\frac{\bar{V}_{i} \bar{V}_{j}}{V D}\right]\left(1-\delta_{i j}\right)+\left[G_{12}+\frac{1}{x_{i}}\left(\frac{\bar{V}_{j}}{D}-V\right)\right] \delta_{i j}
$$

where $\kappa_{T}$ is the isothermal compressibility $\left(k_{B}\right.$ is Boltzmann constant and $T$ the temperature), $\bar{V}_{i}$ is the partial molar volume of species $i, V$ the total volume and $D$ is related to the concentration fluctuations through the expression[43, 46, 47]

$$
D=x_{i}\left(\frac{\partial \beta \mu_{i}}{\partial x_{i}}\right)_{T P}
$$

where $\mu_{i}$ is the chemical potential of species $i(\beta=1 / k T$ is the Boltzmann factor). In practice for dense liquids, the compressibility term is small compared to the others and can be ignored. In addition, we neglect the usually small variations 
with mole fraction of of the partial molar volumes -which amounts to neglect $\partial V^{\text {excess }} / \partial x_{i}$ (the excess volume $V^{\text {excess }}$ is usually at least one order of magnitude smaller than the volumes [48]), and replace these by the volumes of their respective neat systems. This way, the KBI defined in Eq. (4) can be simply related only to the volumes and mole fractions, and the $D$ term needs to be evaluated indirectly. If we write the chemical potention into its usual three different contributions (reference, ideal and excess parts): $\mu_{i}=\mu_{i}^{(0)}+k_{B} T \ln \left(\rho x_{i}\right)+\mu_{i}^{\text {excess }}$, then one gets $[47,46]$

$$
D=1+x_{i}\left(\frac{\partial \beta \mu_{i}^{\text {excess }}}{\partial x_{i}}\right)_{T P}
$$

Under this form, we see that if the excess part becomes independant of the solute concentration, then $D=1$ is reduced solely to its ideal contribution from the chemical potential. One may ask how the excess part may become independant of the solute concentration. By analogy with phase coexistence of a binary mixture, where the chemical potential is the same across the separating interface (by one of the necessary conditions for phase coexistence), then micro-segregation may be considered as a smaller scale version, or rather a local version of phase coexistence. In other words, the condition $D \approx 1$ is consistent with micro-segregation. It does not necessarily imply that that the mixture is ideal, but that concentration fluctuations are negligible despite the micro-heterogeneous configuration. Of course, this condition is not achieved for any micro-heterogeneous mixture, such as in the case of aqueous-alcohol mixtures, for example[47]. We have shown[25] that aqueous-DMSO mixtures behaved as quasi-ideal, due in particular to the fact that almost all water molecules were clustered into small linear aggregates, which acted as supra-molecular entities in order to enforce a quasi-ideality of this particular mixture, as far as the KBI were concerned.

\section{Results}

\subsection{Neat propylamine}

The left side of Fig. 1 shows a snapshot of the N=2048 neat propylamine system, where only the nitrogen and hydrogen atoms are show in filled colored balls, while the methyl united atoms are shown as semi-transparent balls. This way, one can clearly see a number of chain-like patterns formed by the bonding sites. The right side shows a snapshot of the $\mathrm{N}=2048$ neat 1propanol system, where the oxygen atoms are shown in red and the hydrogen in white, with the same convention for the methyl united atoms. This snapshot equally shows visual evidence for chainlike patterns formed by the hydroxyl groups. There is however a big difference between the chain patterns between the two snapshots: those of the propylamine 
system show considerable branching and polydispersity, due to the double hydrogens on the nitrogen site.

Another way to represent the difference between the branched and neat chains in the two systems, is to calculate the cluster distributions formed by the nitrogen and oxygen atoms. This is shown in Fig.2. The cluster distribution of the oxygen atoms of the 1 propanol system shows a clear peak around cluster size 4-5, just like that found for methanol[50, 51], ethanol[28] and tbutanol[50, 51]. Conversely, the cluster distribution of the nitrogen sites of propylamine shows a very weak shoulder about the same cluster size. However, the monomer probability is higher for propylamine than 1propanol. These results are a direct consequence of the visual information of Fig.1: simple chains produce a visible count, while branched chains tend to blur the distribution of aggregated atoms and produce a weak broadened feature instead.

Fig.3a shows the N-N correlations in blue and the N-H correlations in red. The thin and high N-N first peak is typical of the hydrogen bonding between the nitrogen sites, intermediated by the hydrogen sites, just like for water and alcohols[23]. However, unlike water and alcohols, the N-H first correlations are lower than the N-N correlations and show a split two peaks pattern. This is due to the direct $\mathrm{N}-\mathrm{H}$ bonding for the peak at the smaller $\mathrm{r}$ value and the indirect bonding positioned at the same distance as the $\mathrm{N}-\mathrm{N}$ bonding distance through the second hydrogen. These correlations are equally distributed among the two possibilities, which is why they are about twice smaller than the N-N correlations. This observation directly supports the conjectures of the relation between the topology of the solute molecule in relation to its bonding ability. The inset of Fig.3a shows the correlations between the same methyl sites. These correlations are about the same magnitude as the $\mathrm{N}-\mathrm{H}$ ones, and those between the methyl atom closest to the nitrogen atom have the smallest first peak, indicating a least probable contact between these united atoms.

Fig.3b shows particular site-site structure factors, as compared to the pure 1propanol structure factors[49] in the inset. The N-N structure factor shows a prominent pre-peak at $k \approx 0.65 \AA^{-1}$, which corresponds to a structure of approximately $10 \AA$, which is representative of the nitrogen trimer cluster. This corresponds probably to the basic nitrogen aggregates that form the base of the branched chain-like clusters seen in Fig.1. We note that the N-H structure factor equally reveals this cluster pre-peak, almost superimposed to the $\mathrm{N}-\mathrm{N}$ one. The second peak is centered about $k \approx 3 \AA^{-1}$, which corresponds to the Hbonding distance of $r_{H B}=2 \AA$. In contrast, the methyl sites show an ordinary Lennard-Jones type structure factor, with a main peak centered at $k \approx 1.4 \AA^{-1}$ which corresponds to the mean diameter of the methyl site. The oxygen-oxygen (O-O) and oxygenhydrogen $(\mathrm{O}-\mathrm{H})$ 1 propanol structure factors in the inset have a higher pre-peak than the N-N and N-H structure factors of propylamine, which indicates that, for 
the same number and same topology of the methyl sites, the hydrogen bonding difference between straight and branched chains tends to weaken the pre-peak of the latter type of chains. This fact directly supports the arguments developed in the Introduction.

The study of the correlations, both in direct and reciprocal space reveals the Hbonded cluster structure, very much like in alcohols [50, 51], however with the differences induced by the 2 donor hydrogen sites.

\subsection{Aqueous propylamine}

Altough the snapshots are reported for $\mathrm{N}=2048$, all the reported correlations are for the $\mathrm{N}=16000$ systems. This allows better accuracy of the pre-peak features reported below. We studied particularly the propylamine mole fractions $x=0.1$, $0.2,0.5$ and 0.8 .

\subsubsection{Snapshots}

Fig. 4 shows three snapshots of the aqueous propylamine mixtures,taken at the amine mole fractions of $x=0.2,0.5$ and 0.8 . The segregated domain structure is quite obvious in the first two snapshots, with bulky water domains for $x=0.2$ and more linear clusters shaped domains for the equimolar case. These water domains stay very much linear at the highest amine concentration of 0.8 , with few dimers and monomers, as seen by transparency in the last snapshot.

\subsubsection{Cluster distributions}

Fig.5a shows the cluster distribution for propylamine sites (nitrogen in the main panel and the last methyl M3 site in the inset) and Fig.5b shows that for the oxygens of water. Both panels show the distributions for 3 mole fractions of propylamine, $x=0.2$ in red, $x=0.5$ in green and $x=0.8$ in blue. The black curves are for the respective neat systems.

The distribution of large nitrogen site clusters loses its weak shoulder as soon as water is present, and this probability becomes smaller, which indicate a lessened clustering of propylamine with increasing water content. The monomer probability follows the expected rule that their number is larger with smaller propylamine content. The distribution of clusters of the methyl group shows more clustering at small propylamine content $\mathrm{x}=0.2$, which indicates that the oily tails may aggregate to shy away from water and the charged head groups, much like for micelles, but at a smaller scale. Conversely, their distribution tends to remain unchanged at higher propylamine content, indicating that they behave much like in pure propylamine. 
The cluster distribution for the water oxygens shows a trivial trend, with the clustering increased with lesser water content -which is compatible with microheterogeneity- except for the equimolar mixture $\mathrm{x}=0.5$, where the larger cluster size above 5 seem to become prominent than in any other concentrations. This is mostly due to the presence of a large number of chain-like clusters (as seen in the middle snapshot in Fig.4). The fact that the smaller cluster probability of this particular concentration is slightly smaller than the others, indicates that this depletion of this population is compensated by the presence of larger clusters. These differences are however very small, probably indicating a branched structure in the water chains. Indeed, if the water clusters were straight chains, one would probably observe a typical peak such that seen in Fig. 2 for neat 1 propanol. Branching of chains tends to broaden the distribution.

\subsubsection{Site-site correlation functions}

Fig.6 shows the most informative correlation functions of the aqueous propylamine mixture, for the same typical amine concentrations as in Fig.4. The leftmost picture shows the water oxygen-oxygen correlations. It is seen that the first and second neighbour correlations increase with decreasing water content (as observed in the inset which shows a zoom on the pre-peaks). However, a shallow minimum appears beyond the third neighbour correlation, which corresponds to the minimum of the cluster domain correlations, more visible for $x=0.8$, and in agreement with the linear clusters seen in the right snapshot in Fig.4. The shallow minimum corresponds to the minimum of cluster-cluster correlations, which superimpose to the atom-atom correlations. This feature demonstrates the existence of supramolecular water aggregates.

The central panel shows that the water oxygen and amine nitrogen correlations are less important than the water-water correlations, although these correlations tend to show a similar increase with water content decrease. We conclude that that water interacts and form hydrogen bonds preferentially with the surrounding amines when these are in majority. This could explain the presence of water monomer, dimer and small clusters. However, these correlations are seen to be depleted beyond 2 neighbours, indicating the short ranged correlations between antagonist bonding species.

Finally, the rightmost panel shows that the N-N correlations for $x=0.1$ are the smallest of all at first peak level, and that these first peak correlations increase with amine content increase - which is exactly opposite of the behaviour of water. This shows the dramatic difference between Hbonding pattern of water and amine. It is very similar to that we have previously reported in alcohols [28]. We equally observe that the N-N correlations are very small and depleted at contact for $x=0.1$ and $x=0.2$, which shows that the amines tend to Hbond with water rather than 
with themselves.

Incidently, for the $x=0.2$ case, we have compared the correlations of the $\mathrm{N}=16000$ system reported here (in purple) with those of the $\mathrm{N}=2048$ system (in red). It is see that there is almost no influence of the system size as far as short range correlationas are concerned.

\subsubsection{Structure factors}

The structure factors of the water oxygen-oxygen correlation, reported in Fig.7 (left panel) show the typical domain pre-peak correlations that we have previously reported for many other aqueous mixtures. The pre-peak is seen to be more important at high water content for $x=0.2$ (water mole fraction 0.8 ). This is absent for pure water (thin black line). This peak corresponds to the bulky and percolating water domain see in the right panel of Fig.4. As these domains thin out and become linear water clusters, this pre-peak diminishes in proportion. It is interesting that the pure water outer peak of the well known split peak feature [52] - which represents the oxygen-oxygen contact peak, vanishes for any amine content, and only the inner Hbond peak at $k \approx 3 \AA^{-1}$ (corresponding to $r_{H B} \approx 2 \AA$ ) survives, although it decreases with amine content increase. This tends to indicate that the water molecules are less Hbonded at high amine content than at low one, which appears inconsistent with the high first peak of the water correlations in Fig.6 left panel. This apparent discrepancy demonstrates that the increase of the first peak of the $g_{O_{w} O_{w}}(r)$ functions are not only related to direct Hbonding (such as in pure water), but that they also include broader cluster correlations, as clearly shown by the apparent broadening of the first peak in the inset of Fig.6 left panel.

The middle panel shown O-N cross structure factors, which are seen to have both positive and negative pre-peak at the place where $\mathrm{OO}$ have only a positive domain pre-peak. This type of anti-pre-peak is typical of domain segregation, and has beed repeatedly reported in the room temperature ionic liquid literature $[53,54,55]$. This is a very important information in the present context, since it shows that only the case $x=0.2$ shows the anti-pre-peak, when the cross structure factors for $x=0.5$ and 0.8 show positive pre-peaks. This allows us to distinguish between the case where water forms bulky domains (see Fig.4 left panel), which is more synonymous of micro-segregation, from the case where water forms rather linear clusters (as in Fig.4, middle and right panels). This distinction is important, since we argue that water tends to naturally form linear supra-molecules in mixing conditions, and that water segregated domains are often aggregates of such supramolecular linear water clusters[56].

Finally, the rightmost panel shows the N-N structure factors. One sees that, at hight amine content, the pure amine cluster pre-peak (in thin black line -also discussed in Fig. 3 right panel) tends to vanish progressively as the amine content di- 
minishes, while the domain pre-peak emerges (as see at $x=0.2$ and $x=0.1$ ). This latter case corresponds to the bi-continuous segregation of water and propylamine - as seen in snapshot of Fig.4 left panel. The disappearance of the neat amine cluster pre-peak means that propylamine in the domain is more likely Hbonded to water, forming some sort of nitrogen atom interface between the water domains and the alkyl groups.

The influence of the system size dependence is more apparent for the structure factors than for the correlation functions, as seen from the data reported for $x=0.2$ (purple for $\mathrm{N}=16000$ and red for $\mathrm{N}=2048$ ). This difference, even though small, shows that the domain formation is influenced by the system size. However, this difference is not as important as in the case of other mixtures, such as aqueous TBA[23], aqueous acetone[35] or ethanol-benzene[57]. This is attributed to the differences in the micro-heterogeneity and clustering of water, namely the linear clusters found here as opposed to the bulky clusters found in the latter mixtures. Indeed, the distribution of bulky clusters are more likely to be influenced by system size. We observe that the differences in the present context are more important for small $x=0.2$ value, for which bulkier clusters are seen (Fig.4 left panel).

\subsubsection{Kirkwood-Buff integrals}

Since these mixtures look strongly non-ideal, one might expect high values of the KBI. But Fig. 8 shows that the KBI computed from the simulation results are quite small, which is very surprising in view of what is seen for other aqueous mixtures, where the KBI are rather large. We have shown in many cases [58, 59, 23, 35] that the amplitude of the KBI reflect the effective concentration fluctuations. We have plotted the ideal KBI in dashed lines, and Fig. 8 shows that the KBI are nearly ideal in the range $0.3<x<1$. In fact, this is very consistent with the finding reported above. In previous studies, we have underlined that the presence of linear water cluster (supra-molecular water) often leads to small KBI, which means small concentration fluctuations. Indeed, if we try to calculate the D-function from the KBI obtained from the simulations, we obtain the curve shown in the inset, which is equal to 1 in the range $0.5<x<1$. From Eq.(5), this latter information implies that the derivative of the excess chemical potential is zero, which indicates that the chemical potential become independant of the concentration. We interpret this as consistent with water forming tightly bonded supra-molecular entities, which interacts with the rest of the system weakly. This is similar to what we found in benzene-alkane mixtures [57], where $D \approx 1$ was equally found, despite strong molecular interactions. The condition $D=1$ does not mean that there are no interactions, but that the system is homogeneous in terms of clustered or supra-molecular entities. Water specific interactions are strongest in the range 
$0<x<0.3$, as seen from the non-ideal KBI. It is seen that it is the amine-amine $\mathrm{KBI}$ that the most non-ideal, showing that propylamine clustering at small amine content is not trivial. Since $D(x)$ represent the concentration fluctuation term, we see that these are maximal around $x \approx 0.15$, close to the case $x=0.2$ that we have explicitly reported as showing bulky clusters.

\section{Discussion: UCST versus LCST}

As stated in the Introduction, the existence of UCST for aqueous alcohols as opposed to LCST for aqueous amines, can be speculatively understood in terms of the types of water aggregates formed in these different mixtures, namely bulky versus linear aggregates. This is evidently induced by the different type of hydrogen bonding of these respective solute molecules. The main line of argument is that bulky water aggregates have most of their hydrogen bonding sites selfsaturated by water molecules, hence bind less with surrounding solutes than linear water aggregates, which have more open bonding sites to solutes. As a consequence, bulky water aggregates are likely to grow by coalescing, and lead to phase separation under mixing unstability conditions. Such conditions are likely to happen at lower temperatures than higher ones for the simple reason that thermal agitation is likely to increase the disorder of the aggregates rather their coalescence. Hence, aqueous alcohol mixtures with bulky water aggregate domains are likely to have a UCST $[15,16]$. Conversely, the explanation for the existence of a LCST in micro-segregated mixtures seems to be more involved. With that respect, we would like to point out that the currently accepted explanations for the existence of LCST and UCST are essentially related to the seminal paper from Walker and Vause (WV)[60]. While the existence of UCST can be quite simply attributed to mixing under thermal agitation at higher temperature, the WV approach explains remixing at a lower temperature below the LCST as the result of a preferential directional attraction between the two species. This is fully consistent with the hydrogen bond and the experimental fact that LCST exist only in Hbonding mixtures[60]. In this picture, remixing at lower temperature involves essentially the existence of solute-solvent dimers, which overcome the globally unfavourable solute solvent interactions.

However, the VW approach does not account for the existence of microsegregation. Micro-segregation is a stronger principle than a mere solute-solvent directional association. Quite the contrary, it is a local self-segregation of each species, but maintained by cross species interactions across the local domains. Hence, one needs to revisit the WV mixing conditions by taking into account aggregation. The case of the UCST was discussed above at the beginning of the paragraph. We now discuss the case of LCST, in relation to the existence of linear 
water aggregates. In comparison with the difference in bonding between alcohols and amines, namely the lower bonding abillity of amines as discussed in Section 3.1, we hypothetize that linear water aggregates exist because of an appropriate balance between the Hbonding abilities of the solute and water. An increase in temperature will further disfavour the Hbinding ability between water and the amine, due to thermal disorder, leading to increased self-association of water and eventually demixing. Hence, the existence of linear water aggregates is an indirect signature of the nature of the associations between water and the solute, and a possible precursor for an LCST behaviour.

The tentative explanation for the existence of LCST in amine aqueous mixtures with linear water aggregates, while pictorially appealing, needs to be confirmed with other cases. For example, aqueous 2-butoxyethanol mixtures have an LCST[61, 62] while this solute is an alcohol. However, it has an ester group, hence it may favour water-solute interactions more than solute-solute interactions, and induce the presence of linear water aggregates at temperature below its LCST (around 320K). The computer simulations of Gupta and Patey[63, 64] show that demixing consistent with the LCST, appears in the appropriate temperature range, although such simulations require very large system sizes (62000 molecules) in order for the existence of LCST to be settled. We note that the WV picture for LCST is illustrated mainly for binary alcohol mixtures with glycerol as solvent[60]. Since alcohols tend to form chain-like associated structure with their hydroxyl group, it is not excluded that the influence of chains is also an essential mechanism behind the presence of an LCST in these mixtures.

\section{Conclusion}

The study of model aqueous propylamine mixtures reveals specificities of water segregation that we have observed in several different contexes. In the range $0<x<0.3$ we find that water forms bulky domains, similar to that we have observed in aqueous alcohols [24, 23], which lead to a prominent domain pre-peak in the oxygen-oxygen structure factor. In contrast, in the range $0.3<x<1$, water forms linear clusters, similar to what we found in water-DMSO mixtures[25], and leading to modest structure factor pre-peaks and near ideal KBI. Hence, the aqueous propylamine behaves in a composite manner, between aqueous alcohols at low solute content and aqueous DMSO at high solute content. In fact, despite high structure factor pre-peak at small solute content, this system has quite moderate KBI, which indicates that it is nearly ideal, and consequently acts as a well mixed and homogeneous system in terms of supra-molecular entities. This is an interesting system, which seems nearly ideal, despite very strong Hbonding interactions, but which tend to self-segregate each species into weakly interacting 
macro-molecular entities, and probably more the water than the amine molecules.

The discussion above opens several questions about the kinetics of the water aggregation under mixing conditions. What is the exchange time constant of monomeric water molecules between the clusters entities? How does this kinetic influences the dynamical properties rather than the static properties. We do not provide answers to these questions in this work. Beyond these questions, the interpretation proposed here deserves a deeper investigation, perhaps to eventually lead to a better understanding of the well documented, but still not deeply understood phenomena such as the hydrophobic effect, and the role of water in the biophysical context.

\section{Acknowledgments}

M. Požar thanks both the Croatian Science Foundation for financial support under the project 4514 "Multi-scale description of meso-scale domain formation and destruction", and the French Embassy in Croatia for financial support through "bourse du Gouvernement Français".

\section{References}

[1] G.T. Rochelle, Science 325, 1652 (2009)

[2] A.B. Rao and E.S. Rubin, Environ. Sci. Technol. 36, 4467-4475 (2002)

[3] C. Ruiz-Capillas and F. Jimenez-Colmenero, Critical Reviews in Food Science and Nutrition, 44:7-8, 489-599 (2005)

[4] M.H. Silla Santos, International Journal of Food Microbiology 29, 213-231 (1996)

[5] D. Hallifax and J.B. Houston, Drug Metabolism and Disposition, 34:18291836 (2006)

[6] L. Edvinsson, C. Owman and N.-O. Sjolberg, Brain Research 115, 377-393 (1976)

[7] F.F. Kadlubar, M.A. Butler, K.R. Kaderlik, H.C. Chou, and N.P. Lang, Environmental Health Perspectives 98: 69-74 (1992)

[8] R.J. Turesky and L. Le Marchand, Chem Res Toxicol 15; 24(8): 1169-1214 (2011) 
[9] A. Babusyte, M. Kotthoff, J. Fiedler and D. Krautwurst, Journal of leukocyte biology 93.3, 387-394 (2013)

[10] S. Dixit, J. Crain, W. C. Poon, J. L. Finney and A. K. Soper, Nature 416, 829 (2002)

[11] T. Yamaguchi, K. Hidaka and A. K. Soper, Mol. Phys. 96, 1159 (1999).

[12] M. Tomšič, A. Jamnik, G. Fritz-Popovski, O. Glatter and L. Vlcek, J. Phys.Chem. B111, 1738 (2007)

[13] W. L. Jorgensen, J. Tirado-Rives, J. Am. Chem. Soc. 110, 1657 (1988)

[14] R.M. Stephenson, J. Chem. Eng. Data 38, 625-629 (1993)

[15] Y. Aoki and T. Moriyoshi, J. Chem. Thermo. 101173 (1978)

[16] Y. Aoki and T. Moriyoshi, J. Chem. Thermo. 7537 (1975)

[17] G.A. Orozco, C. Nieto-Draghi, A.D. Mackie and V. Lachet, J. Phys. Chem. B 115 (49), pp 14617?14625 (2011)

[18] C. Oostenbrink, A. Villa, A. E. Mark, W. F. van Gunsteren, J. Comput.Chem. $25,1656-1676$ (2004)

[19] C. Oostenbrink, D. Juchli and W.F. van Gunsteren, ChemPhysChem 6, 1800$1804(2005)$

[20] W.L. Jorgensen, D.S. Maxwell and J. Tirado-Rives, J. Am. Chem. Soc. 118, 11225-11236 (1996)

[21] R.C. Rizzo and W.L. Jorgensen, J. Am. Chem. Soc. 121, 4827-4836 (1999)

[22] P. G. Kusalik, D. Berman and A. Laaksonen, J. Chem. Phys. 113, 8036 (2000).

[23] B. Kežić and A. Perera, J. Chem. Phys. 137, 014501 (2012)

[24] M. Mijaković, B. Kežić, L. Zoranić, F. Sokolić, A. Asenbaum, C. Pruner, Emmerich Wilhelm and A. Perera, Journal of Molecular Liquids 164, 66 ?73 (2011)

[25] A. Perera and R. Mazighi, J Chem Phys. 143, 154502 (2015)

[26] A. Perera, Pure Appl. Chem. 88189 (2016) 
[27] J.P. Hansen and I.R. McDonald, Theory of Simple Liquids (Academic, London, 1986)

[28] M. Požar, B. Lovrinčević, L. Zoranić, T. Primorac, F. Sokolić and A. Perera, Phys. Chem. Chem. Phys. 18, 23971 (2016)

[29] C. Tanford "The hydrophobic effect: formation of micelles and biological membranes". New York: Wiley (1973)

[30] W. Kauzmann W, Advances in Protein Chemistry. 14 1-63 (1959)

[31] B. Widom, P. Bhimalapuram and K. Koga, Phys. Chem. Chem. Phys. 53058 (2003)

[32] D. Chandler, Nature (London) 437640 (2005)

[33] P. Wiggins, Plos one, 1 e1406 (2008)

[34] P. Ball, Chem. Rev. 10874 (2008)

[35] B. Kežić and A. Perera, J. Chem. Phys. 137, 134502 (2012)

[36] D. van der Spoel., E. Lindahl, B. Hess., G. Groenhof, A. E. Mark and H. J.C. Berendsen, 2005, J.Comp. Chem. 26, 1701

[37] J.C. Berendsen, J.P.M. Postma, W.F. Von Gusteren and J. Hermans, in Intermolecular Forces, edited by B. Pullman (Reidel, Dordrecht, 1981)

[38] S. Nose, Mol. Phys. 52:255-268 (1984)

[39] W.G. Hoover, Phys. Rev. A 31:1695-1697 (1985)

[40] M. Parrinello and A. Rahman, J. Appl. Phys. 52, 7182 (1981)

[41] J. M. Martínez and L. Martínez, Journal of Computational Chemistry, 24(7):819-825, 2003; ibid L. Martínez, R. Andrade, E. G. Birgin, J. M. Martínez. Journal of Computational Chemistry, 30(13):2157-2164, 2009

[42] E. Oran Brigham, The Fast Fourier Transform (Prentice Hall, 1988)

[43] J.G. Kirkwood and F. Buff, J. Chem. Phys.19, 774 (1950)

[44] A. Perera and F. Sokolić, J. Chem. Phys. 121 (22), 11272 (2004)

[45] J.L. Lebowitz, J.K. Percus, Phys. Rev. 122,1675 (1961)

[46] A. Ben-Naim, J. Chem. Phys. 67, 4884 (1977) 
[47] E. Matteoli and L. Lepori, J. Chem. Phys. 80, 2856 (1984)

[48] A. Perera, F. Sokolic, L. Almasy, Y. Koga J. Chem. Phys. 124, 124515 (2006)

[49] B. Kežić and A. Perera, J. Chem. Phys, 135 , 237104 (2011)

[50] A. Perera, F. Sokolić and L. Zoranić, Physical Review E 75, 060502(R) (2007)

[51] L. Zoranić, F. Sokolić and A. Perera, J. Chem. Phys. 127: 024502 (2007)

[52] A.Perera, Molecular Physics 109, 20, 2433-2441 (2011)

[53] H. V. R. Annapureddy, H. K. Kashyap, P. M. De Biase and C. J. Margulis, J. Phys. Chem. B114, 16838 (2010)

[54] A. Triolo, O. Russina, H.-J. Bleif, E. Di Cola, J. Phys. Chem. B111 4641 (2007).

[55] C.S. Santos, H.V.R. Annapureddy, N.S. Murthy, H.K. Kashyap, E.W. Castner, C.J. Margulis, J. Chem. Phys. 134064501 (2011)

[56] M. Požar, A. Kerasidou, B. Lovrinčević, M. Mijaković, T. Primorac, L. Zoranić, F. Sokolić, V. Teboul and A. Perera, J. Chem. Phys 145, 144502 (2016)

[57] M. Požar, J.B Seguier, J. Guerche, R. Mazighi, L. Zoranić, M. Mijaković, B. Kežić-Lovrinčević, F. Sokolić and A. Perera, Phys. Chem. Chem. Phys., 17, 9885 (2015)

[58] A. Perera, L. Zoranić, F. Sokolić and R. Mazighi, Journal of Molecular Liquids 159, 52 (2011)

[59] M. Mijaković, K.D. Polok, B. Kežić, F. Sokolić, A. Perera and L. Zoranić, Molecular Simulation (2014) DOI: 10.1080/08927022.2014.923567

[60] J. S. Walker and C. A. Vause, J. Chem. Phys. 79, 2660 (1983)

[61] Y. Koga, J. Phys. Chem. 96, 10467 (1992)

[62] A. G. Aizpiri, F. monroy, C. del Campo, R. G. Rubio and M. Diaz Pena, Chem. Phys. (North Holland) 16531 (1992)

[63] R. Gupta and G. N. Patey, J. Phys. Chem B115, 15323 (2011)

[64] R. Gupta and G. N. Patey, J. Mol. Liq. 177, 102 (2013) 


\section{FIGURE CAPTIONS}

- Fig.1 - Snapshot of neat propylamine for system size $\mathrm{N}=2048$. The nitrogen sites are shown in blue, the hydrogen sites in white and the methyl group united atom sites in semi-transparent cyan.

- Fig.2- Cluster distribution probabillities for the nitrogen atoms of propylamine in blue and in red for the oxygen atoms of 1 propanol.

- Fig.3 - (a) Selected site-site correlation functions. Nitrogen-nitrogen correlations in blue, nitrogen-hydrogen in red. The inset shows selected methylmethyl correlations(see text): M1-M1 in magenta, M2-M2 in orange and M3-M3 in brown. (b) Sites-site structure factor corresponding to some of the site-site correlations shown in (a) with the same color codes. The inset shows the structure factors for neat 1-propanol[49] (O-O in blue, O-H in red and M2-M2 in orange)

- Fig.4 - Snapshot of the aqueous propylamine mixtures for 3 different propylamine mole fractions: left for $x=0.2$, middle for $x=0.5$ and right for $x=0.8$. Water molecules oxygen and hydrogen atoms are shown in red and white, respectively, propylamine molecules are shown in dark blue, except for the right panel where they are shown in semi-transparent blue.

- Fig.5 - Cluster distribution probabilities versus cluster size for (a) the nitrogen sites in the left panel (last methyl site in the inset) and (b) the oxygen sites in the right panel. Red curves are for propylamine concentration $x=0.2$, green for $x=0.5$ and blue for $x=0.8$. The black lines are for the pure systems.

- Fig.6 - Selected site-site correlation functions of the equimolar aqueous for different propylamine mole fractions : $x=0.1$ in jade, $x=0.2$ in purple (red a for the $\mathrm{N}=2048$ system), $x=0.5$ in blue and $x=0.8$ in cyan; pure water (left panel) and pure propylamine (right panel) in black . Left, middle and right panels correspond to for the oxygen-oxygen, oxygen-nitrogen and nitrogen-nitrogen correlations, respectively.

- Fig.7 - Selected site-site structure factors corresponding to the correlation functions shown in Fig.6 with the same color and panel codes.

- Fig.8 - Kirkwood-Buff integrals of the aqueous-propylamine mixtures. Color code is as follows, blue for water-water, magenta for propylamine-propylamine and green for the cross KBI. Open squares are the KBI from the present simulations, full lines correspond to the D-coefficient shown in the inset (see text) and dashed lines are the ideal KBI. 
a) Propylamine

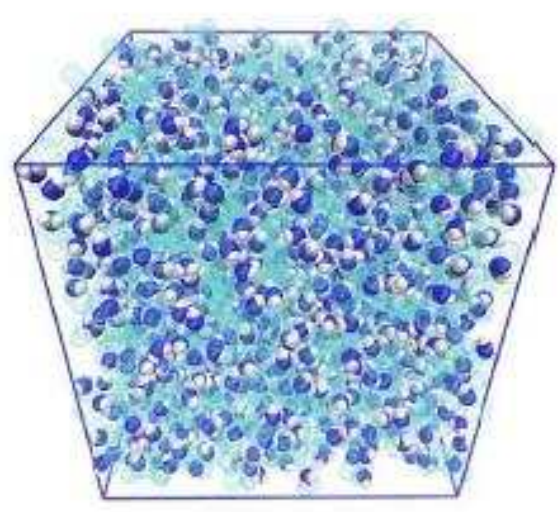

b) 1propanol

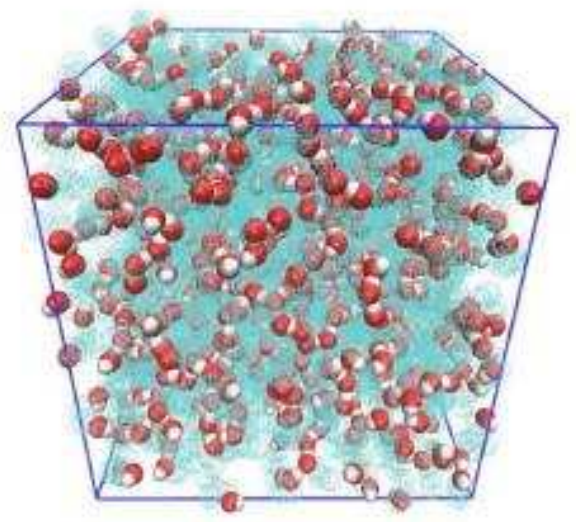

Figure 1

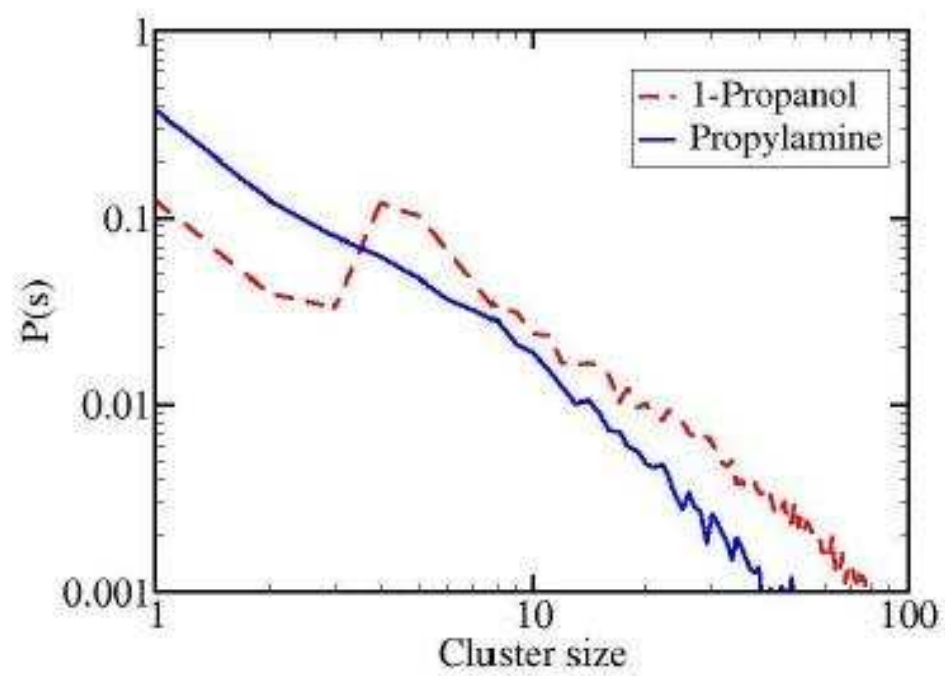

Figure 2 
(a)

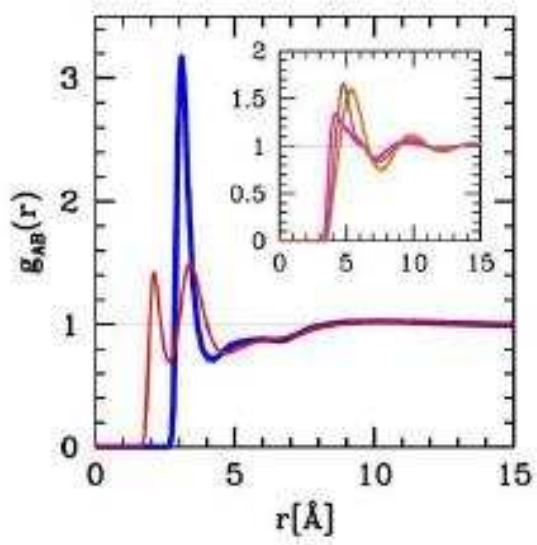

(b)

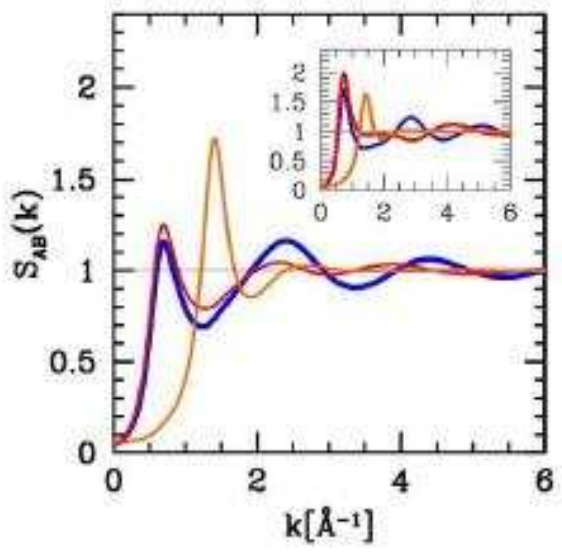

Figure 3

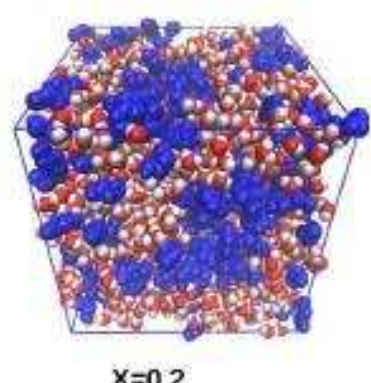

$x=0.2$

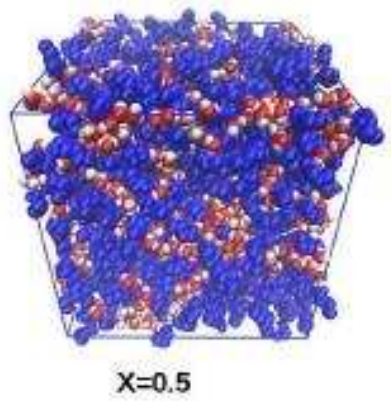

$x=0.5$

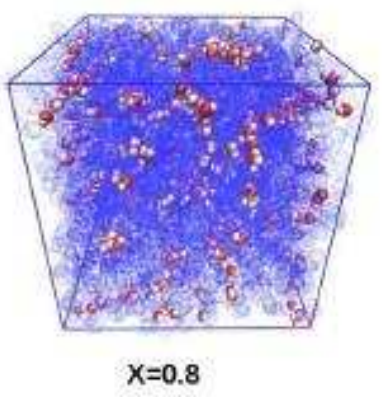

$x=0.8$

Figure 4 
a) Propylamine

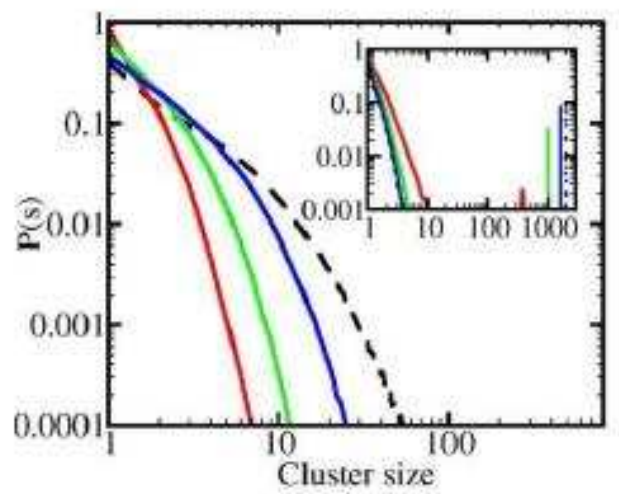

b) Water

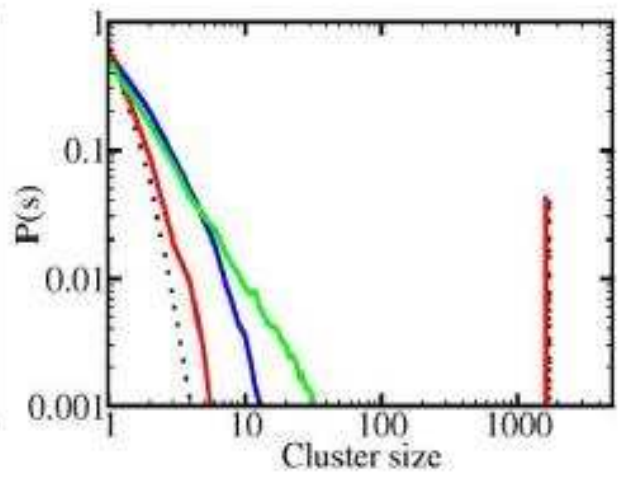

Figure 5
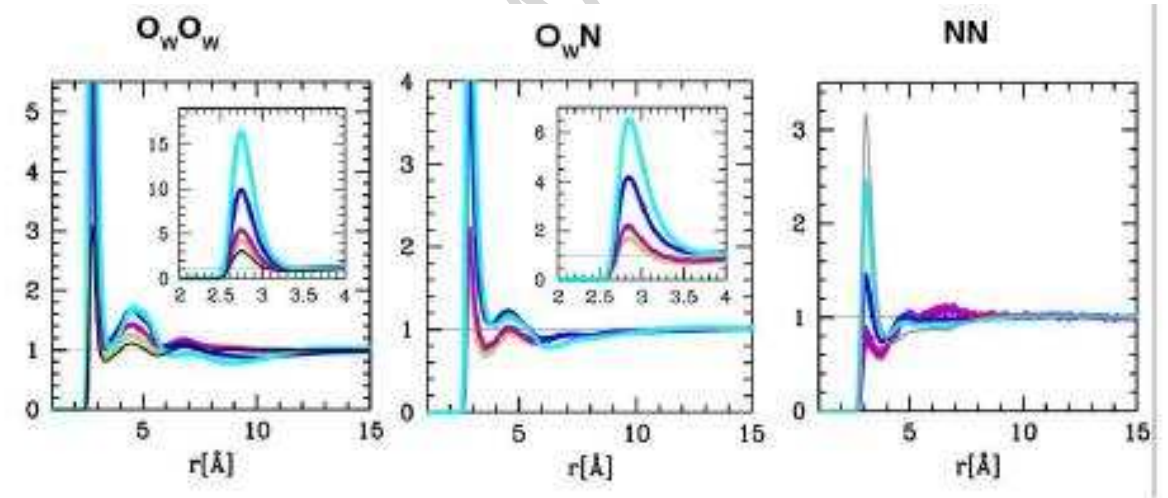

Figure 6
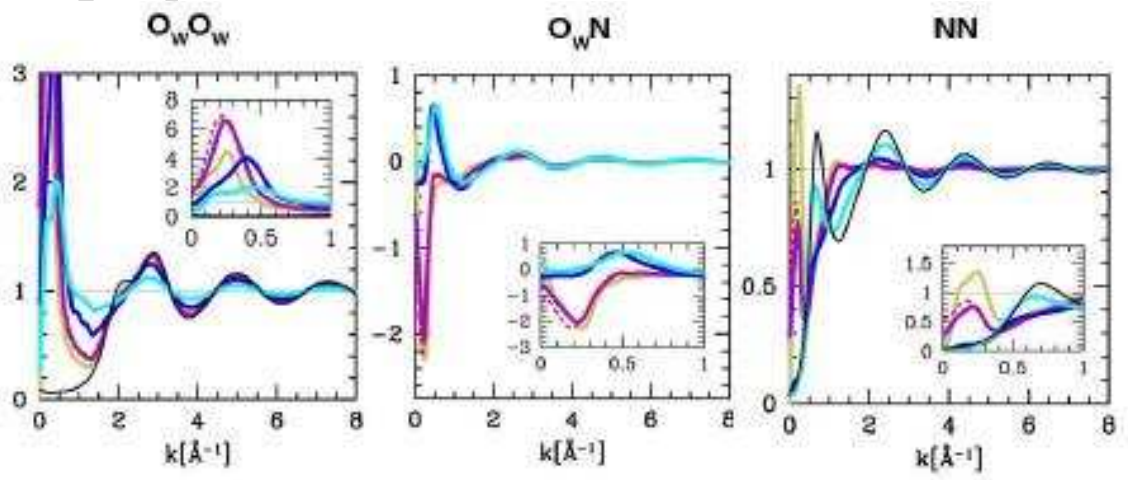

Figure 7 


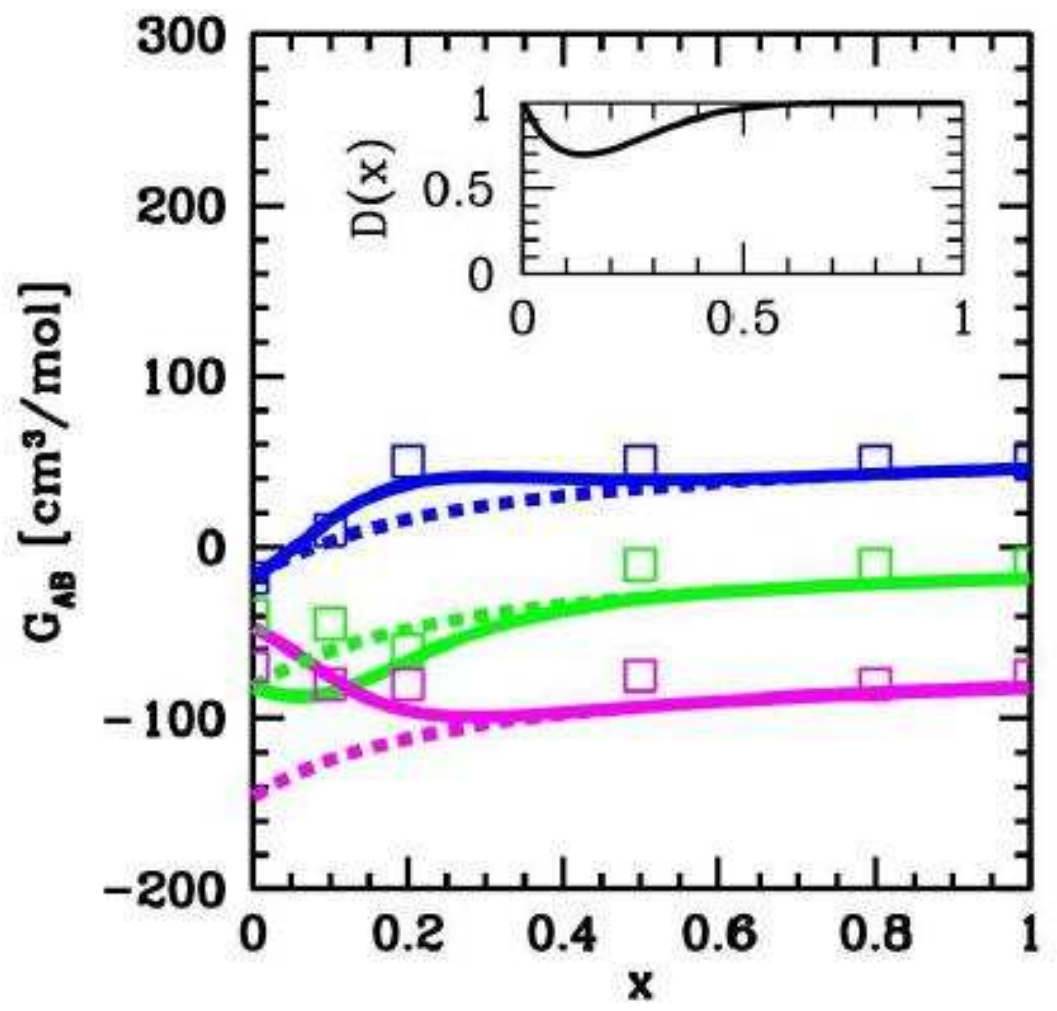

Figure 8 


\section{Highlights}

- Molecular dynamics study of aqueous propylamine

- Hydrogen bonding of pure proplymine versus 1propanol

- How the molecular topology of amine molecule affect the structure of aqueous mixture as compared with similar alcohol molecule (propylamine versus propanol)

- Water forms linear aggregates with propylamine, versus bulky aggregates in 1propanol

- Why aqueous-amines have LCST when compared with absence in aqueous-alcohol 